\title{
Pirfenidone for idiopathic pulmonary fibrosis: analysis of pooled data from three multinational phase 3 trials
}

\author{
Paul W. Noble ${ }^{1}$, Carlo Albera², Williamson Z. Bradford ${ }^{3}$, Ulrich Costabel ${ }^{4}$, \\ Roland M. du Bois ${ }^{5}$, Elizabeth A. Fagan ${ }^{3}$, Robert S. Fishman ${ }^{3}$, Ian Glaspole ${ }^{6}$, \\ Marilyn K. Glassberg ${ }^{7}$, Lisa Lancaster ${ }^{8}$, David J. Lederer ${ }^{9}$, Jonathan A. Leff ${ }^{3}$, \\ Steven D. Nathan ${ }^{10}$, Carlos A. Pereira ${ }^{11}$, Jeffrey J. Swigris ${ }^{12}$, \\ Dominique Valeyre ${ }^{13}$ and Talmadge E. King $\mathrm{Jr}^{14}$
}

Affiliations: ${ }^{1}$ Cedars-Sinai Medical Center, Los Angeles, CA, USA. ${ }^{2}$ University of Turin, Turin, Italy. ${ }^{3}$ InterMune Inc., Brisbane, CA, USA. ${ }^{4}$ Ruhrlandklinik, University of Duisburg-Essen, Essen, Germany. ${ }^{5}$ Imperial College, London, UK. ${ }^{6}$ Alfred Hospital and Monash University, Melbourne, Australia. ${ }^{7}$ University of Miami Miller School of Medicine, Miami, FL, USA. ${ }^{8}$ Vanderbilt University Medical Center, Nashville, TN, USA. ${ }^{9}$ Columbia University Medical Center, New York, NY, USA. ${ }^{10}$ Inova Fairfax Hospital, Falls Church, VA, USA. ${ }^{11}$ Paulista School of Medicine, Federal University of São Paulo, São Paulo, Brazil. ${ }^{12}$ National Jewish Health, Denver, CO, USA.

${ }^{13}$ Assistance Publique-Hôpitaux de Paris, Avicenne University Hospital, Bobigny, France. ${ }^{14}$ University of California, San Francisco, San Francisco, CA, USA.

Correspondence: Paul W. Noble, Department of Medicine, Cedars-Sinai Medical Center, NT Suite 2119, 8700 Beverly Blvd, Los Angeles, CA 90048, USA. E-mail: Paul.Nobledacshs.org

ABSTRACT Pirfenidone is an antifibrotic agent that has been evaluated in three multinational phase 3 trials in patients with idiopathic pulmonary fibrosis (IPF). We analysed pooled data from the multinational trials to obtain the most precise estimates of the magnitude of treatment effect on measures of disease progression.

All patients randomised to pirfenidone $2403 \mathrm{mg}^{-\mathrm{day}^{-1}}$ or placebo in the CAPACITY or ASCEND studies were included in the analysis. Pooled analyses of outcomes at 1 year were based on the prespecified end-points and analytic methods described in the ASCEND study protocol.

A total of 1247 patients were included in the analysis. At 1 year, pirfenidone reduced the proportion of patients with $\mathrm{a} \geqslant 10 \%$ decline in per cent predicted forced vital capacity or death by $43.8 \%$ (95\% CI 29.3-55.4\%) and increased the proportion of patients with no decline by 59.3\% (95\% CI 29.0-96.8\%). A treatment benefit was also observed for progression-free survival, 6-min walk distance and dyspnoea. Gastrointestinal and skin-related adverse events were more common in the pirfenidone group, but rarely led to discontinuation.

Analysis of data from three phase 3 trials demonstrated that treatment with pirfenidone for 1 year resulted in clinically meaningful reductions in disease progression in patients with IPF.

@ERSpublications

Treatment with pirfenidone for 1 year results in clinically meaningful reductions in IPF disease progression http://ow.ly/StvBk

For editorial comments see Eur Respir J 2016; 47: 27-30 [DOI: 10.1183/13993003.01669-2015].

This article has supplementary material available from erj.ersjournals.com

Received: Jan 082015 | Accepted after revision: Sept 012015

Clinical trial: This study is registered at www.clinicaltrials.gov with identifier numbers NCT01366209, NCT00287729 and NCT00287716.

Support statement: This study was sponsored by InterMune Inc. (Brisbane, CA, USA). Funding information for this article has been deposited with FundRef.

Conflict of interest: Disclosures can be found alongside the online version of this article at erj.ersjournals.com

Copyright OERS 2016. ERJ Open articles are open access and distributed under the terms of the Creative Commons Attribution Non-Commercial Licence 4.0 


\section{Introduction}

Pirfenidone is an oral antifibrotic agent that has been evaluated in three similar multinational phase 3 trials in patients with idiopathic pulmonary fibrosis (IPF) [1,2]. The CAPACITY trials (studies 004 and 006) [1] evaluated the efficacy and safety of treatment with pirfenidone for a minimum of 72 weeks; the ASCEND trial (study 016) [2] evaluated treatment with pirfenidone for 52 weeks. The pre-specified primary efficacy end-point in all three trials was the change from baseline in per cent predicted forced vital capacity (FVC \% pred).

Evaluation of outcomes in the CAPACITY trials showed that pirfenidone significantly reduced the decline in FVC \% pred at week 72 in study 004, but not in study 006 [1], prompting a third multinational phase 3 trial (ASCEND) to confirm the effect of pirfenidone in patients with IPF. In the ASCEND trial, pirfenidone significantly reduced the decline from baseline to week 52 in FVC \% pred and improved progression-free survival [2], confirming observations from the CAPACITY studies as well as two prior Japanese studies and an independent meta-analysis of data from the CAPACITY and Japanese studies conducted under the auspices of the Cochrane Collaboration [3-5]. Analysis of outcomes at 1 year in each of the multinational phase 3 studies provided evidence of a pirfenidone treatment benefit on multiple measures of disease progression, including FVC \% pred, 6-min walk distance (6MWD) and progression-free survival $[1,2]$.

To obtain more precise estimates of the magnitude of treatment effect in the broad population of patients enrolled in these trials and further evaluate the effect of pirfenidone in subpopulations of interest, we conducted an exploratory analysis of pooled data from the ASCEND and CAPACITY studies. Analyses were based on the pre-specified end-points and analytic methods described in the ASCEND study protocol. Outcomes were assessed at 1 year (the duration of the ASCEND study) to allow each study to contribute equal follow-up times to the landmark analyses. The magnitude of effect beyond 1 year was also evaluated at the time of the primary end-point in the respective studies (week 52 in ASCEND and week 72 in CAPACITY).

\section{Methods}

Study subjects

Source data included all patients randomised to treatment with pirfenidone $2403 \mathrm{mg} \cdot \mathrm{day}^{-1}$ or placebo in either the CAPACITY [1] or the ASCEND studies [2]. The phase 2 and phase 3 Japanese trials of pirfenidone were not included in this analysis because they differed from the three multinational trials in study design and end-point selection. The study population in the Japanese trials was $100 \%$ Japanese. In addition, the Japanese trials had higher patient drop-out rates and more missing data than the multinational trials. Finally, certain outcome measures in the multinational trials were assessed differently compared with the Japanese trials, including FVC and progression-free survival $[3,4]$.

Eligibility criteria for the CAPACITY and ASCEND studies were similar, but not identical $[1,2]$. Briefly, eligibility for the CAPACITY studies required a confident diagnosis of IPF by the local investigator, FVC $\%$ pred $\geqslant 50 \%$, per cent predicted diffusing capacity of the lung for carbon monoxide (DLCO \% pred) $\geqslant 35 \%$, either FVC \% pred or DLCO \% pred $\leqslant 90 \%$, forced expiratory volume in $1 \mathrm{~s}$ (FEV1)/FVC ratio $\geqslant 0.70$ and $6 \mathrm{MWD} \geqslant 150 \mathrm{~m}$. Eligibility for the ASCEND study required a centrally confirmed diagnosis of IPF according to the radiological and histopathological criteria of the American Thoracic Society/European Respiratory Society/Japanese Respiratory Society/Latin American Thoracic Association [6], FVC \% pred $\geqslant 50 \%$ and $\leqslant 90 \%$, DLCO $\%$ pred $\geqslant 30 \%$ and $\leqslant 90 \%$, FEV $1 / \mathrm{FVC}$ ratio $\geqslant 0.80$, and $6 \mathrm{MWD} \geqslant 150 \mathrm{~m}$.

\section{Study design}

The primary objectives of the analysis were to obtain the most precise estimates of the magnitude of the pirfenidone treatment effect in patients with IPF, and to further interrogate the effect of pirfenidone in subpopulations defined on the basis of demographics and baseline measures of disease status.

In each of the phase 3 trials, eligible patients were randomly assigned to oral pirfenidone $2403 \mathrm{mg} \cdot \mathrm{day}^{-1}$ or $^{-}$ matched placebo. Study drug was administered with food in three equally divided doses and escalated to the full dose over 2 weeks. Clinical efficacy parameters were assessed at 12-week intervals in the CAPACITY studies and 13-week intervals in the ASCEND study. In the pooled analysis, clinical efficacy outcomes are reported at 3-month intervals from baseline to month 12 . Safety outcomes are based on analysis of data at week 52 in all three studies.

\section{Statistical analysis}

The statistical analysis plan for the pooled analysis was finalised prior to unblinding of results from the ASCEND study. Pooled efficacy analyses were based on the pre-specified primary and secondary end-points from the ASCEND study, analysed according to the methodology described in the ASCEND statistical analysis plan [2]. The primary efficacy analysis in the ASCEND study was the change from 
baseline to week 52 in FVC \% pred, analysed in the intent-to-treat population using a ranked analysis of covariance (ANCOVA) model $[7,8]$. The magnitude of treatment effect was estimated by comparing the distribution of patients in the pirfenidone and placebo groups across two clinically meaningful thresholds: $10 \%$ absolute decline in FVC \% pred or death, or no decline in FVC \% pred (change in FVC \% pred $\geqslant 0$ ). Results for all landmark analyses are reported as the point estimate and 95\% confidence interval for the distribution of patients in the pooled pirfenidone and placebo groups across categorical thresholds of change from baseline to month 12, as defined in the ASCEND study protocol. Inferential statistics are reported for the purpose of informing assessments of the variability of the treatment effect. Supportive analyses were also conducted to assess the robustness of the effect of treatment on FVC; these included the mean change from baseline to month 12 in FVC and the cumulative distribution of FVC change from baseline to month 12 .

Secondary efficacy analyses included the change from baseline to month 12 in $6 \mathrm{MWD}$, change from baseline to month 12 in dyspnoea (as measured by the University of California San Diego Shortness of Breath questionnaire (UCSD SOBQ)) and progression-free survival (time to the first occurrence of death or disease progression, defined as a confirmed $\geqslant 10 \%$ decline in FVC $\%$ pred or a confirmed $\geqslant 50 \mathrm{~m}$ decline in 6MWD). Mortality outcomes in the pooled population from the ASCEND and CAPACITY studies have been reported previously, and are therefore not included in the current report.

For the rank ANCOVA analyses, missing values due to death were assigned the worst ranks based on the time of death from randomisation. Missing values for reasons other than death were imputed with the average value from three patients with the smallest sum of squared differences at each visit with nonmissing data. For the analysis of mean change from baseline in FVC, missing values due to death were assigned the worst possible value (i.e. "0").

Interactions between treatment and subgroups defined on the basis of demographics and baseline measures of disease status were evaluated for the change from baseline to month 12 in FVC \% pred in the pooled population using rank ANCOVA analyses with treatment-by-baseline characteristic term in the model. Each interaction was tested individually without correction for multiplicity. Additionally, interactions between treatment and study were assessed using rank ANCOVA analyses with treatment-by-study term in the model; p-values $<0.05$ indicate statistical evidence of an interaction. To assess the overall effect of treatment across studies, the relative risk or hazard ratio and $95 \%$ confidence interval were calculated for each clinical efficacy end-point in the pooled population.

The magnitude of treatment effect was also evaluated in a pooled analysis of outcomes at the time of the study primary end-point in the respective trials (week 52 in ASCEND and week 72 in CAPACITY). To account for differences in trial duration between the ASCEND and CAPACITY studies, a Cox proportional hazards model was used to estimate the hazard ratio and $95 \%$ confidence interval for each clinical efficacy end-point, including time to confirmed decline in FVC $\geqslant 10 \%$ or death, confirmed decline in $6 \mathrm{MWD}$ $\geqslant 50 \mathrm{~m}$ or death, confirmed increase in UCSD SOBQ score $\geqslant 20$ points or death and progression-free survival time. The model was stratified by study and by geographic region within study (USA and rest of world) and data were censored at the time of the study primary end-point in the respective study (day 365 in ASCEND study and day 505 in CAPACITY). Differences between treatment groups were evaluated using the log-rank test.

Safety outcomes are reported as treatment-emergent events, defined as events occurring between baseline and 28 days after the last dose of study drug up to 1 year, and summarised descriptively. Adverse events were coded to preferred terms in the Medical Dictionary for Regulatory Activities, version 11.0.

\section{Results}

A total of 1247 patients were included in the analysis; of these, 623 were randomised to treatment with pirfenidone $2403 \mathrm{mg} \cdot \mathrm{day}^{-1}$ and 624 were randomised to treatment with placebo in either the ASCEND or CAPACITY studies.

Demographics and baseline characteristics were generally well balanced across studies and treatment groups (table 1). Median baseline values for FVC \% pred and DLCO \% pred were slightly lower, and median time since diagnosis was slightly longer, among patients enrolled in ASCEND compared with the CAPACITY studies; however, there was considerable overlap in these and other measures of baseline disease status across studies (see online supplementary figure S1).

A total of 1122 (90.0\%) patients in the pooled population completed 12 months of treatment (566 (90.9\%) patients in the pirfenidone group versus 556 (89.1\%) in the placebo group). Study treatment was discontinued prematurely in $97(15.6 \%)$ patients in the pirfenidone group and $81(13.0 \%)$ patients in the placebo group (see online supplementary table S1 for patient disposition in the individual studies). 
TABLE 1 Demographics and baseline characteristics

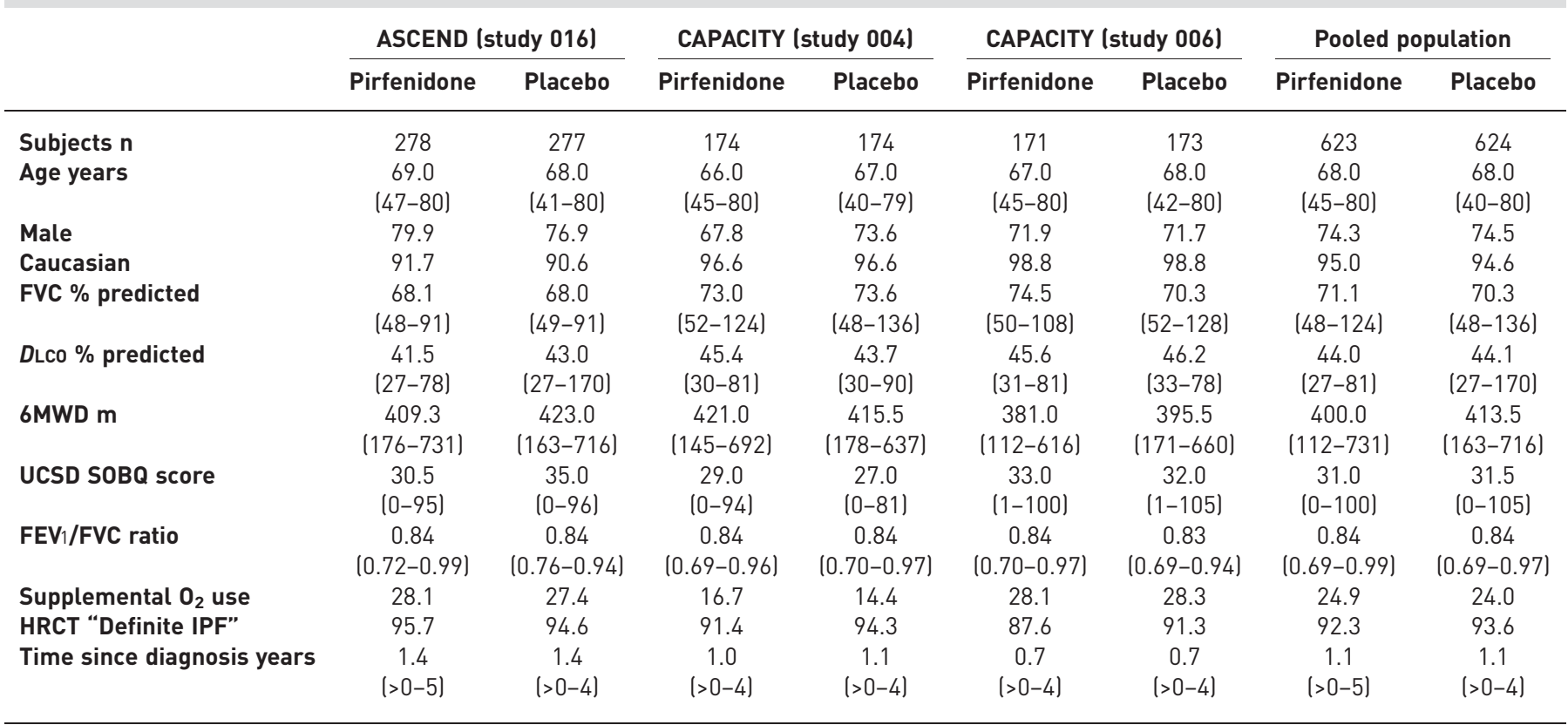

Data presented as median (range) or \%, unless otherwise stated. FVC: forced vital capacity; DLco: diffusing capacity of the lung for carbon monoxide; 6MWD: 6-min walk distance; UCSD SOBQ: University of California San Diego Shortness of Breath Questionnaire; FEV1: forced expiratory volume in $1 \mathrm{~s}$; HRCT: high-resolution computed tomography; IPF: idiopathic pulmonary fibrosis.

\section{Efficacy outcomes}

Analysis of outcomes at 1 year demonstrated that pirfenidone reduced the proportion of patients with a $\geqslant 10 \%$ decline in FVC \% pred or death by 43.8\% (95\% CI 29.3-55.4) and increased the proportion of patients with no decline by 59.3\% (95\% CI 29.0-96.8) compared with placebo (rank ANCOVA, p<0.001). The treatment benefit was evident at each time point beginning with the first assessment at 3 months (figure 1a). Sensitivity analyses using alternative imputation methods for missing data showed that the magnitude of treatment effect on the change in FVC at month 12 was highly consistent across analyses (see online supplementary table S3). Additionally, a favourable treatment effect was observed across the full distribution of change from baseline to 1 year in FVC \% pred (figure 1b). For each point along the cumulative distribution of change from baseline, FVC outcomes favoured pirfenidone over placebo. The mean change from baseline to 1 year in FVC was $-216 \mathrm{~mL}$ in the pirfenidone group and $-363 \mathrm{~mL}$ in the placebo group (absolute difference $148 \mathrm{~mL}$, relative difference 40.7\%; $<<0.001$; figure $1 \mathrm{c}$ ). Consistent with the categorical analysis, the treatment effect emerged by the first FVC assessment at month 3 and persisted throughout the duration of observation.

Subgroup analysis of FVC outcomes showed a consistent magnitude of treatment effect across all strata for each demographic variable and baseline measure of disease status (figure 2). The standardised treatment effect and 95\% confidence interval for the change from baseline to 1 year favoured pirfenidone and excluded "no effect" for all subgroups. With the possible exception of time since IPF diagnosis (unadjusted $\mathrm{p}=0.034$ ), there was no statistical evidence of significant interaction between treatment and baseline demographics or measures of disease status.

In the pooled analysis of change from baseline to 1 year in $6 \mathrm{MWD}$, pirfenidone reduced the proportion of patients with $\mathrm{a} \geqslant 50 \mathrm{~m}$ decline or death by $28.7 \%$ (95\% CI 15.1-40.2) compared with placebo. A total of $153(24.8 \%)$ patients in the pirfenidone group experienced $\mathrm{a} \geqslant 50 \mathrm{~m}$ decline in 6MWD or death compared with $214(34.8 \%)$ patients in the placebo group ( $<<0.001$; figure $3 a)$. Additionally, pirfenidone reduced the risk of death or disease progression at 1 year by $38 \%$ compared with placebo (hazard ratio $0.62,95 \%$ CI $0.51-0.75 ; \mathrm{p}<0.001$; figure $3 \mathrm{~b}$ ). Fewer patients in the pirfenidone group compared with placebo experienced a qualifying event for each component of the composite end-point, including a confirmed $\geqslant 10 \%$ decline in FVC $\%$ pred $(6.7 \%$ versus $13.6 \%)$, a confirmed $\geqslant 50 \mathrm{~m}$ decline in $6 \mathrm{MWD}$ (16.9\% versus $20.7 \%)$ and death (3.0\% versus $5.1 \%)$. Finally, pooled analysis of dyspnoea outcomes showed that fewer patients in the pirfenidone group compared with placebo experienced $a \geqslant 20$-point increase in the UCSD SOBQ score or death at month $12(148(24.0 \%)$ patients in the pirfenidone versus $194(31.4 \%)$ patients in the placebo group; relative difference $23.7 \%$, 95\% CI 8.4-36.4; $\mathrm{p}=0.047$; figure 3c). 
a)

ASCEND (study 016)

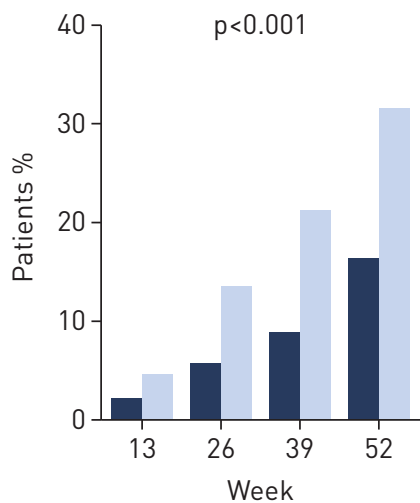

Pirfenidone ( $\mathrm{n}=278$ )

Placebo ( $n=277$ )
CAPACITY (study 004)

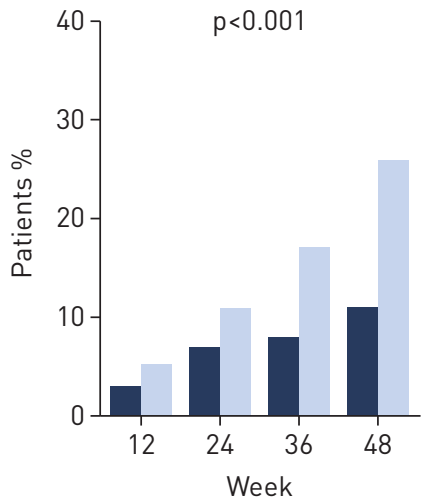

Pirfenidone ( $n=174)$

Placebo $(n=174)$
CAPACITY (study 006)

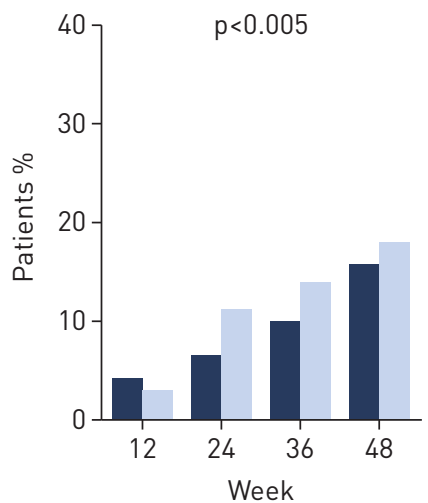

Pirfenidone $(n=171)$

Placebo $(n=173)$
Pooled population

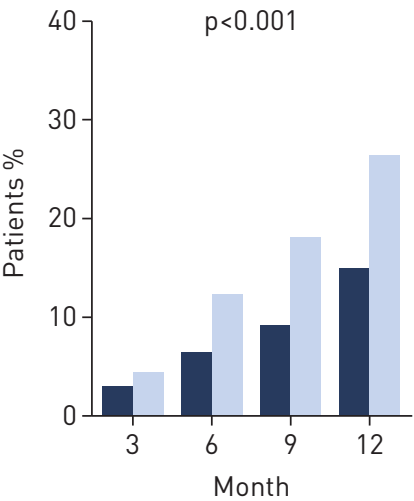

Pirfenidone $(\mathrm{n}=623)$

- Placebo $(n=624)$
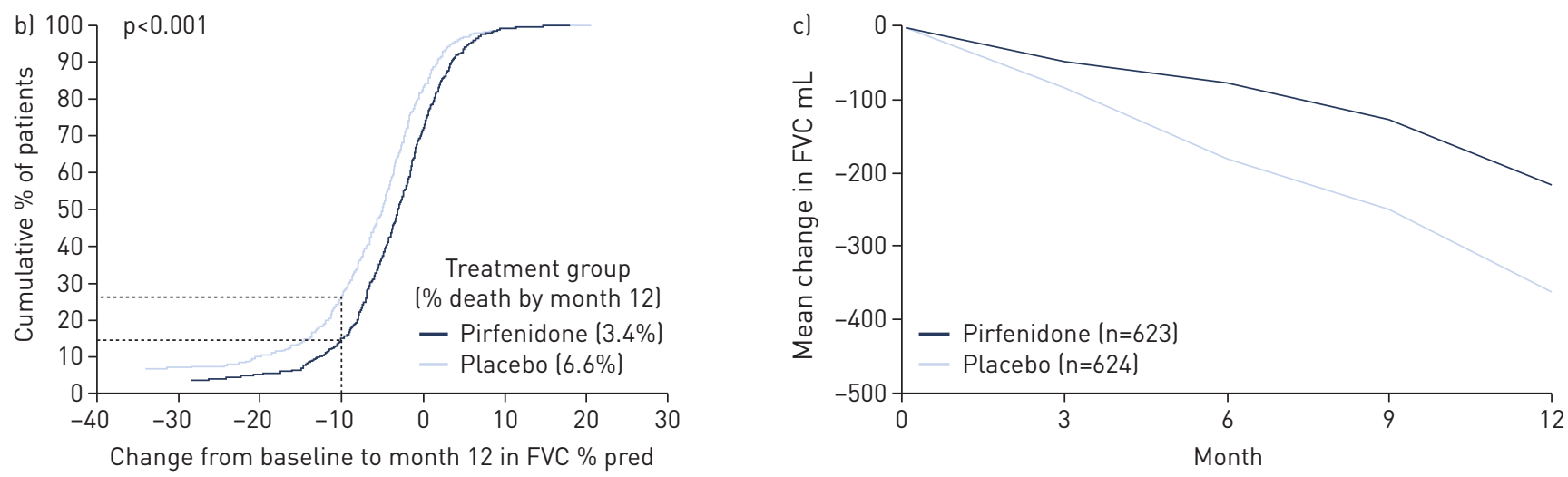

\begin{tabular}{|r|c|c|c|c|}
\hline Absolute difference $\mathrm{mL}$ & 36 & 104 & 123 & 148 \\
\hline Relative difference $\%$ & 43.5 & 57.3 & 49.1 & 40.7 \\
\hline Rank ANCOVA p-value & $<0.001$ & $<0.001$ & $<0.001$ & $<0.001$ \\
\hline
\end{tabular}

FIGURE 1 FVC outcomes. a) Proportion of patients with forced vital capacity (FVC) $\%$ predicted decline $\geqslant 10 \%$ or death. Rank ANCOVA (pirfenidone $2403 \mathrm{mg} \cdot$ day $^{-1}$ versus placebo). b) Cumulative distribution of change from baseline to month 12 in FVC \% pred in the pooled population (n=1247). c) Mean change from baseline in FVC in the pooled population. Assessed at weeks 12, 24, 36 and 48 in CAPACITY [1] and weeks 13, 26, 39 and 52 in ASCEND [2].

Figure 4 summarises the overall efficacy results in the pooled population. The point estimate and $95 \%$ confidence interval for the relative risk/hazard ratio favoured pirfenidone and excluded "no effect" for each clinical efficacy outcome. Additionally, analysis of outcomes by study revealed generally similar treatment effects across all three independent studies, with no statistical evidence of an interaction between treatment and study (figure 5).

Assessment of outcomes at the time of the study end-point in the respective studies showed that the magnitude of treatment effect following treatment for up to 72 weeks was generally consistent with the observed magnitude of effect at 1 year (figure 6). The risk of $a \geqslant 10 \%$ decline in FVC $\%$ pred or death was reduced by $52 \%$ (hazard ratio $0.48,95 \%$ CI $0.37-0.63 ; \mathrm{p}<0.001$ ), the risk of death or disease progression was reduced by $38 \%$ (hazard ratio $0.62,95 \%$ CI $0.52-0.75 ; \mathrm{p}<0.001$ ) and the risk of $\mathrm{a} \geqslant 50 \mathrm{~m}$ decrement in $6 \mathrm{MWD}$ or death was reduced by $34 \%$ (hazard ratio $0.66,95 \%$ CI $0.54-0.82 ; \mathrm{p}<0.001$ ) in the pirfenidone group compared with placebo. Dyspnoea outcomes suggested a more modest treatment benefit; at the time of the study primary end-point, there was a $25 \%$ reduction in the risk of worsening dyspnoea (as measured by $\mathrm{a} \geqslant 20$-point increase in UCSD SOBQ score) or death in the pirfenidone group compared with placebo (hazard ratio 0.75 , 95\% CI $0.60-0.93$; $\mathrm{p}=0.007$ ).

\section{Safety outcomes}

Consistent with previous studies in patients with IPF, nearly all patients in both treatment groups experienced at least one adverse event during the 1-year observation period $(98.7 \%$ and $96.5 \%$ in the pirfenidone and 
Subgroup

Region

Age years

Sex

Race/ethnicity

FVC \% pred

Dıco \% pred

$\begin{aligned} & 40-<50 \\ & \geqslant 50\end{aligned}$

6MWD m $\quad 0-350$

$\begin{aligned} & 350-<450 \\ & \geqslant 450\end{aligned}$

$\mathrm{FEV}_{1} / \mathrm{FVC} \quad<0.80$

Supplemental $\mathrm{O}_{2}$ use

Smoker status

Time since diagnosis years
$0.80-<0.85$

$\geqslant 0.85$

USA

Rest of world

$65-74$

$\geqslant 75$

Female

White

Non-white

$<65$

$65-\leqslant 80$

$>80$

Yes

No

Current/former Never

$<1$

$1-\leqslant 2$

$>2$
Favours placebo Favours pirfenidone

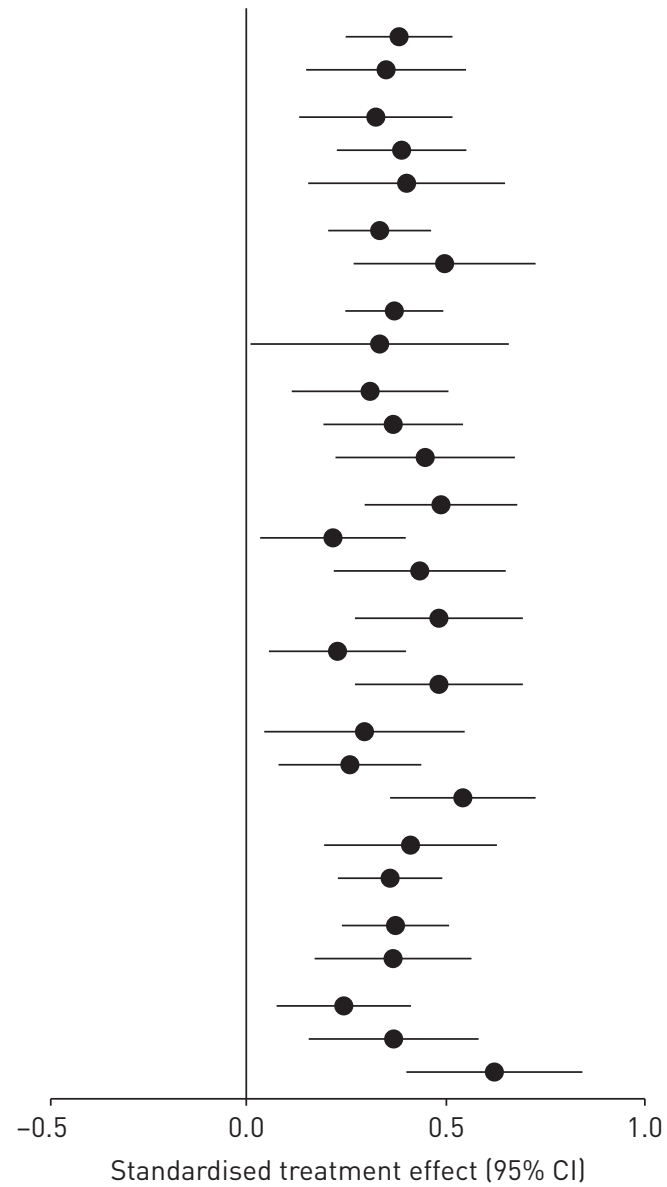

FIGURE 2 Subgroup analysis of change in forced vital capacity (FVC) \% predicted at 1 year in the pooled population. DLCO: diffusing capacity of the lung for carbon monoxide; 6MWD: 6-min walk distance; FEV 1 : forced expiratory volume in $1 \mathrm{~s}$. $p>0.05$ for all tests for interaction between treatment and subgroup except time since diagnosis (nominal $p=0.034$; not corrected for multiple comparisons).

placebo groups, respectively). More patients in the pirfenidone group compared with placebo experienced an adverse event that led to early discontinuation of treatment $(74(11.9 \%)$ versus $54(8.7 \%))$; however, there were fewer serious adverse events (128 (20.5\%) versus 139 (22.3\%)) and fewer treatment-emergent deaths (14 (2.2\%) versus $32(5.1 \%)$ ) in the pirfenidone group compared with placebo (see online supplementary table S2).

The most common adverse events are summarised in table 2. Gastrointestinal and skin-related adverse events occurred more frequently in the pirfenidone group than the placebo group. Consistent with prior observations, these were generally mild to moderate in severity and infrequently led to premature discontinuation of treatment.

Elevations in aminotransferases $\geqslant 3$ times the upper limit of normal occurred in $3.2 \%$ of patients in the pirfenidone group compared with $0.6 \%$ of patients in the placebo group, including one patient in the pirfenidone group with concurrent elevation in serum total bilirubin $>2$ times the upper limit of normal. All such elevations were reversible and without clinical sequelae.

Safety outcomes according to baseline measures of disease severity are summarised in table 3 . In both the pirfenidone and placebo groups, patients with more severe physiologic impairment at baseline had a higher incidence of grade 3 and grade 4 adverse events, serious adverse events, and adverse events leading to treatment discontinuation. However, there was no evidence of a more pronounced effect of disease severity on the risk of adverse events in the pirfenidone group compared with placebo.

\section{Discussion}

IPF is a heterogeneous disease with markedly variable rates of disease progression [9]. As a result, precise estimation of the magnitude of treatment effect on measures of disease progression is challenging, 
ASCEND (study 016)

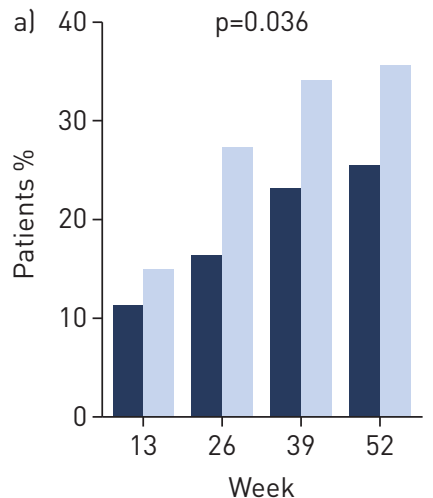

Pirfenidone ( $\mathrm{n}=278)$

Placebo $(n=277)$

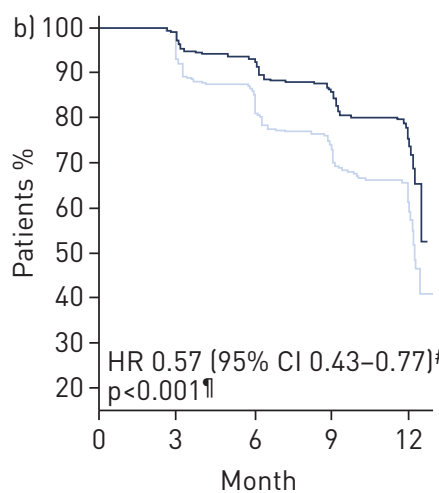

- Pirfenidone ( $\mathrm{n}=278$ )

- Placebo ( $n=277)$

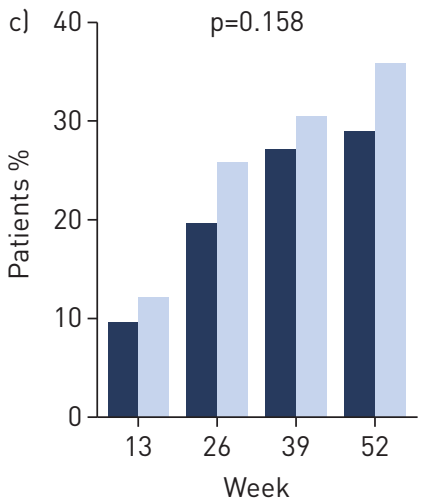

Pirfenidone $(\mathrm{n}=278)$

Placebo ( $n=277)$
CAPACITY (study 004)

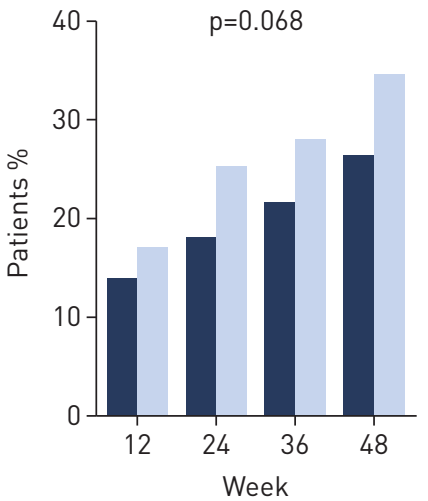

Pirfenidone ( $n=174)$

Placebo $(n=174)$

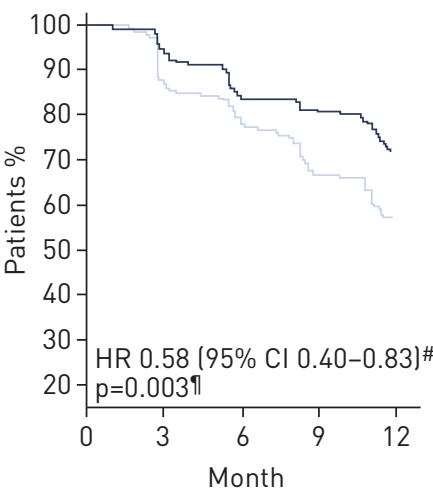

- Pirfenidone ( $\mathrm{n}=174)$

- Placebo $(n=174)$

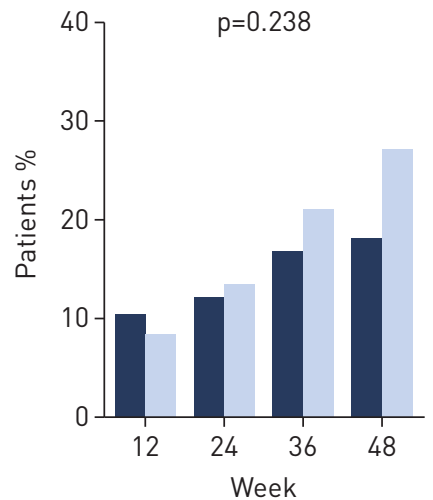

Pirfenidone ( $n=174)$

Placebo $(n=174)$
CAPACITY (study 006)

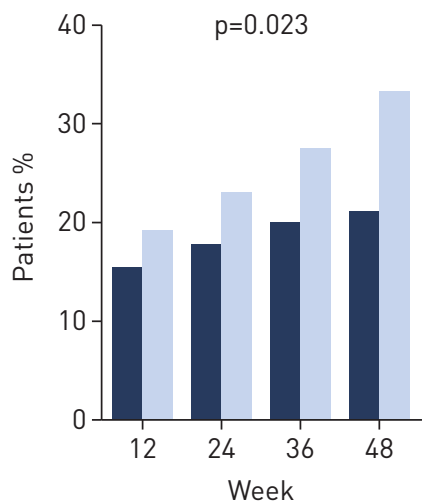

Pirfenidone $(n=171)$

Placebo ( $n=173$ )

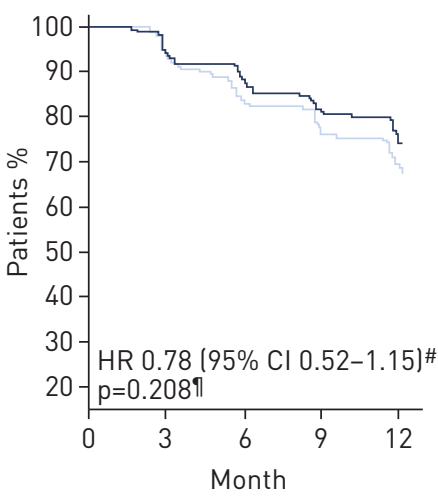

- Pirfenidone $(n=171)$

- Placebo (n=173)

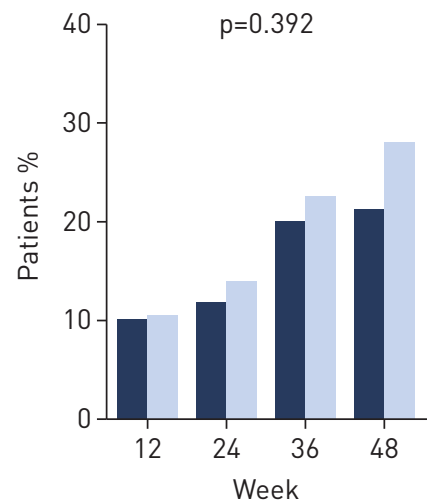

Pirfenidone ( $n=171)$

Placebo ( $n=173$ )
Pooled population

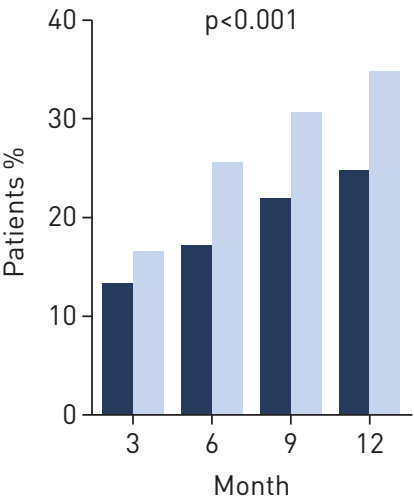

Pirfenidone $(n=623)$

Placebo (n=624)

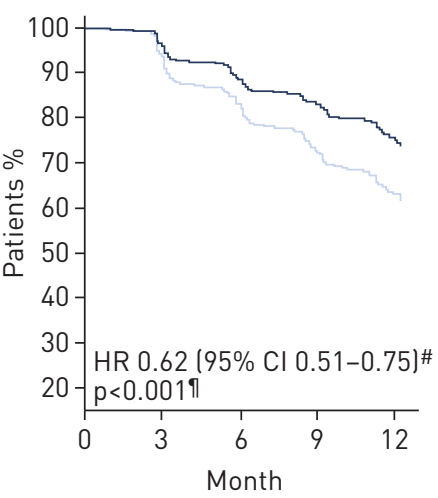

- Pirfenidone ( $n=623$ )

- Placebo ( $n=624)$

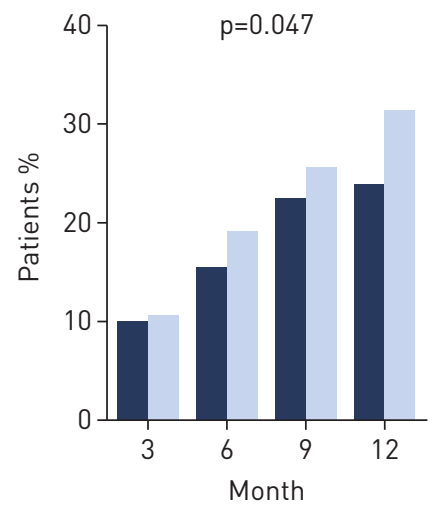

Pirfenidone ( $\mathrm{n}=623)$

Placebo $(n=624)$

FIGURE 3 Secondary outcomes. a) Proportion of patients with a decline in 6 -min walk distance $\geqslant 50 \mathrm{~m}$ or death. Rank ANCOVA (pirfenidone $2403 \mathrm{mg} \cdot$ day $^{-1}$ versus placebo). b) Progression-free survival at 1 year. Time to death or disease progression (confirmed $\geqslant 10 \%$ decline in forced vital capacity $\%$ predicted or $\geqslant 50 \mathrm{~m}$ decline in 6-min walk distance). c) Proportion of patients with an increase in UCSD SOBQ SCore $\geqslant 20$ points or death. Rank ANCOVA (pirfenidone $2403 \mathrm{mg} \cdot$ day $^{-1}$ versus placebo). HR: hazard ratio; UCSD SOBQ: University of California San Diego Shortness of Breath Questionnaire (scale 0-120; higher scores indicate worse dyspnoea). \#: Cox proportional hazards model; " : log-rank test.

particularly in individual clinical trials of moderate size. Analysis of pooled data from multiple clinical trials with similar study designs, patient populations and outcomes allows for more precise estimation of the magnitude of effect associated with a given treatment [10]. 


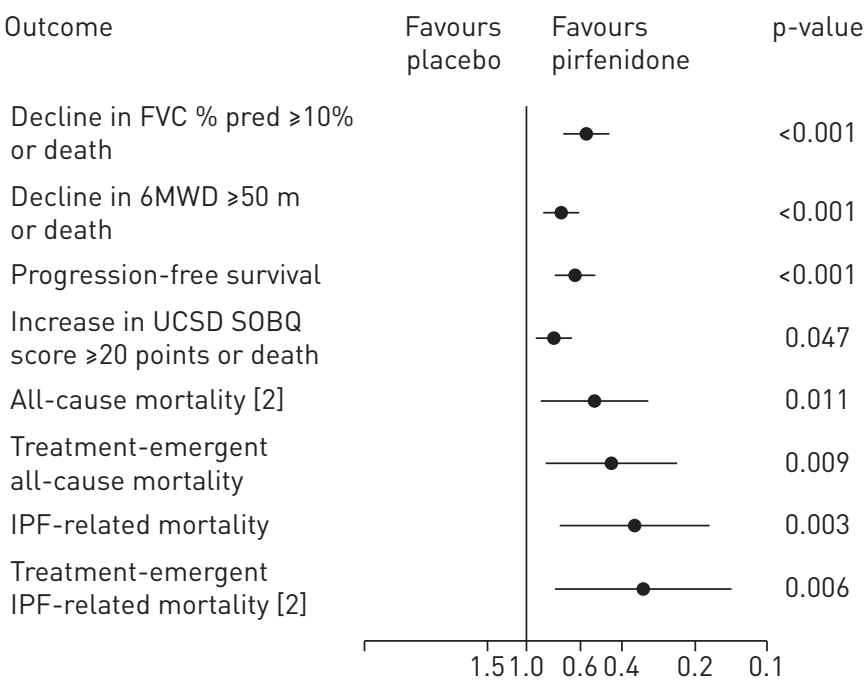

Relative risk/hazard ratio $(95 \% \mathrm{Cl})$

FIGURE 4 Summary of clinical efficacy outcomes at 1 year in the pooled population ( $n=1247)$. FVC: forced vital capacity; 6MWD: 6-min walk distance; UCSD SOBQ: University of California San Diego Shortness of Breath Questionnaire; IPF: idiopathic pulmonary fibrosis.

In the present analysis, we used pooled data from three similar phase 3 trials evaluating pirfenidone in patients with IPF to derive the most precise estimates to date of the magnitude of treatment effect on measures of pulmonary function and disease status. Our results demonstrate that treatment with pirfenidone for 1 year reduced the proportion of patients with $\mathrm{a} \geqslant 10 \%$ decline in FVC $\%$ pred or death by $43.8 \%$ and improved progression-free survival by $38 \%$ compared with placebo. Additionally, $28.7 \%$ fewer patients in the pirfenidone group compared with placebo experienced $\mathrm{a} \geqslant 50 \mathrm{~m}$ decrement in $6 \mathrm{MWD}$ or death. Analysis of outcomes following treatment for up to 72 weeks yielded similar results; for each end-point, the magnitude of treatment effect was consistent with the observed magnitude of effect at 1 year.

FVC \% pred\#

$$
\begin{aligned}
& \text { Favours Favours } \\
& \text { placebo pirfenidone }
\end{aligned}
$$

\section{CAPACITY $(004)$}

CAPACITY (006)

Pooled population

6MWDף

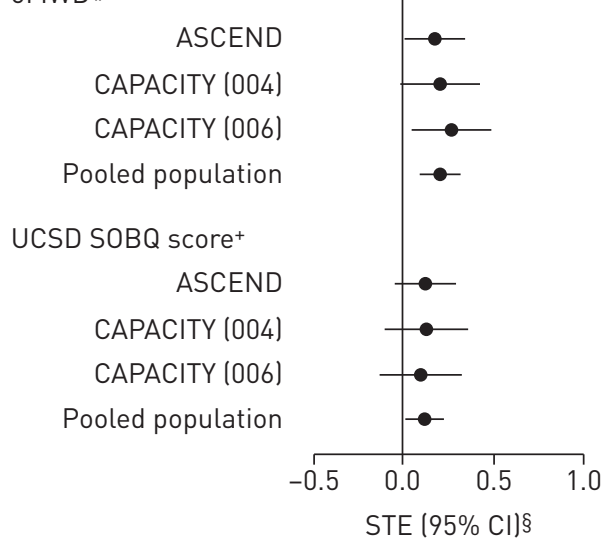

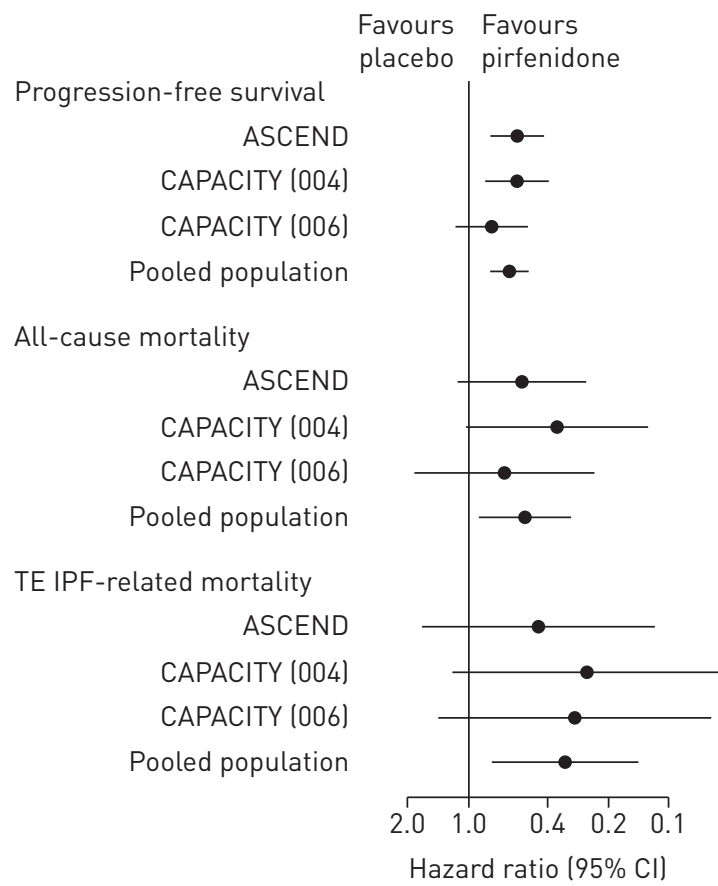

Favours Favours lacebo pirfenidone

FIGURE 5 Summary of clinical efficacy outcomes at 1 year across studies. FVC: forced vital capacity; 6MWD: 6-min walk distance; UCSD SOBQ: University of California San Diego Shortness of Breath Questionnaire; TE: treatment-emergent; IPF: idiopathic pulmonary fibrosis; STE: standardised treatment effect. $p>0.05$ for all tests for interaction between treatment and study. ${ }^{\#}$ : decline in FVC $\%$ predicted $\geqslant 10 \%$ or death; ${ }^{~}$ : decline in $6 \mathrm{MWD} \geqslant 50 \mathrm{~m}$ or death; ${ }^{+}$: increase in UCSD SOBQ score $\geqslant 20$ points or death; ${ }^{\S}$ : estimated as the difference in the rank mean change from baseline between groups divided by the estimate of the common standard deviation from each study. 

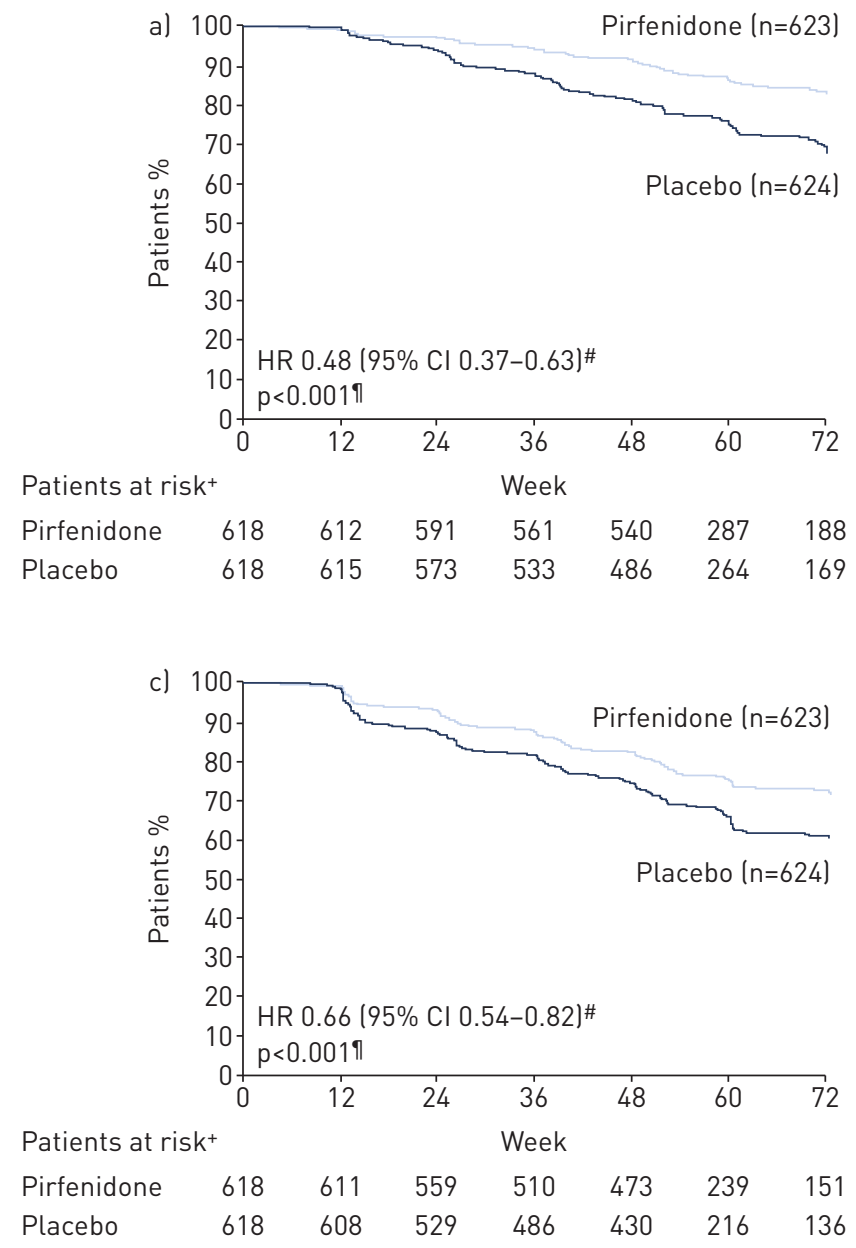

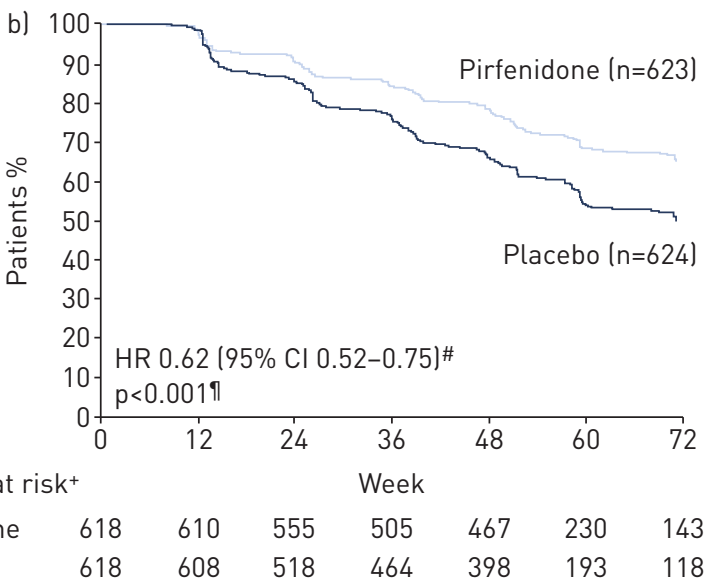

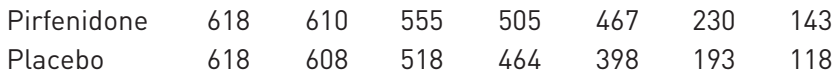

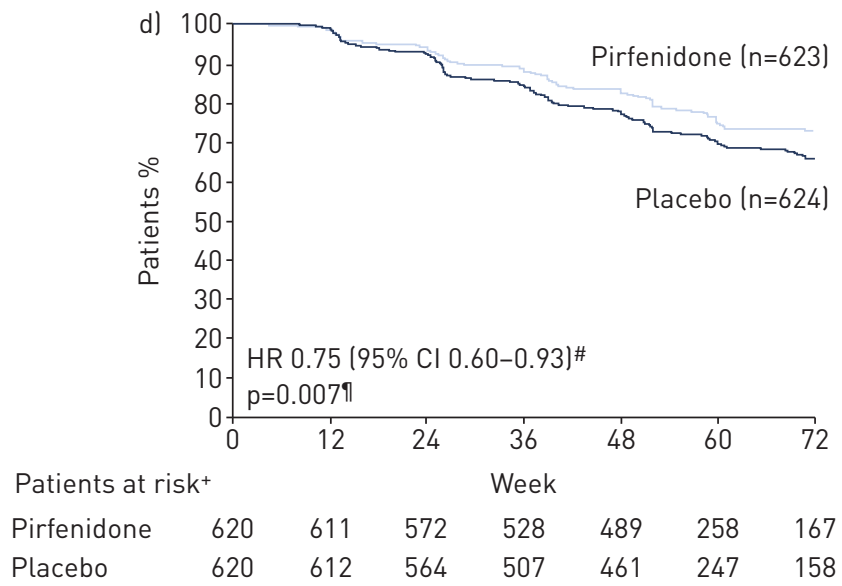

FIGURE 6 a) Kaplan-Meier distribution of time to confirmed $\geqslant 10 \%$ decline in forced vital capacity (FVC) $\%$ predicted or death in the pooled population. b) Kaplan-Meier distribution of progression-free survival in the pooled population. Time to first occurrence of death or disease progression (confirmed $>10 \%$ decline in FVC \% pred or confirmed $>50$ m decrement in 6MWD). c) Kaplan-Meier distribution of time to confirmed $\geqslant 50 \mathrm{~m}$ decline in 6MWD or death in the pooled population. d) Kaplan-Meier distribution of time to worsening dyspnoea or death in the pooled population. Defined as a confirmed $\geqslant 20$-point increase in UCSD SOBQ score or death. HR: hazard ratio; UCSD SOBQ: University of California San Diego Shortness of Breath Questionnaire (scale 0-120; higher scores indicate worse dyspnoea). \# : Cox proportional hazards model; " : log-rank test. ${ }^{+}$: reported as the number of patients at risk on the last day of each 12-week study period in each study lexcludes five patients from the pirfenidone group and six patients from the placebo group who had no post-randomisation follow-up assessment).

Analysis of FVC change in subgroups defined on the basis of demographics and baseline measures of disease status showed that the magnitude of the pirfenidone treatment benefit was also consistent across subpopulations. For each subgroup, the standardised treatment effect favoured pirfenidone and the 95\% confidence interval excluded "no effect". Additionally, the cumulative distribution of FVC change from baseline to 1 year in the pooled pirfenidone and placebo groups showed a consistent treatment benefit across the full spectrum of longitudinal change in FVC. Collectively, these findings demonstrate that the pirfenidone treatment benefit is observed across the continuum of baseline disease severity and subsequent rates of FVC progression.

Safety outcomes in the pooled population were consistent with the known safety profile of pirfenidone. Gastrointestinal and skin-related events were the most commonly reported adverse events in the pirfenidone group; these were typically mild to moderate in severity and rarely led to treatment discontinuation. While adverse events were generally more common in patients with more severe physiologic impairment, there was no evidence of a more pronounced effect of physiologic impairment on the risk of adverse events in the pirfenidone group compared with placebo.

The analysis of pooled data from multiple clinical studies is a widely used method of deriving more precise estimates of the magnitude of treatment effect on outcomes of interest, including those that occur with a low frequency in the individual studies [10]. Additionally, pooling study results can increase the generalisability of the findings by demonstrating the effect of an intervention in a broader patient population [11]. Finally, pooling data from multiple studies increases the power to assess the effect of an intervention in 
TABLE 2 Treatment-emergent adverse events at 1 year ${ }^{\#}$

\begin{tabular}{lcc} 
& Pirfenidone & Placebo \\
\hline Subjects n & 623 & 624 \\
Nausea & 35.5 & 15.1 \\
Cough & 23.1 & 24.0 \\
Diarrhoea & 24.6 & 18.8 \\
Upper respiratory tract infection & 22.6 & 20.2 \\
Fatigue & 23.0 & 16.8 \\
Headache & 20.5 & 18.1 \\
Rash & 29.2 & 9.0 \\
Nasopharyngitis & 15.1 & 15.9 \\
Dyspnoea & 13.2 & 16.0 \\
Dizziness & 16.7 & 10.1 \\
Dyspepsia & 17.8 & 6.7 \\
Bronchitis & 11.1 & 13.1 \\
Idiopathic pulmonary fibrosis & 8.5 & 14.4 \\
Vomiting & 12.7 & 6.1 \\
Anorexia & 12.4 & 4.3 \\
Gastro-oesophageal reflux disease & 10.3 & 5.6 \\
\hline
\end{tabular}

Data are presented as $\%$ unless otherwise stated. ${ }^{*}$ : occurring in $\geqslant 10 \%$ of patients in either treatment group (sorted by total incidence across treatment groups).

subpopulations of interest. By way of illustration, eligibility criteria for the ASCEND study required a centrally confirmed diagnosis of IPF and a baseline DLCO $\%$ pred $\geqslant 30 \%$, while the CAPACITY studies required an IPF diagnosis determined by site investigators and a baseline DLCO $\%$ pred $\geqslant 35 \%$. Pooling data from ASCEND and CAPACITY allowed for estimation of the magnitude of treatment effect in the broader population and increased the power to interrogate potential differential effects of treatment across subgroups.

TABLE 3 Subgroup analysis of safety outcomes at 1 year

\begin{tabular}{|c|c|c|c|c|c|c|c|c|c|}
\hline \multicolumn{5}{|c|}{ Pirfenidone $2403 \mathrm{mg} \cdot \mathrm{day}^{-1}$} & \multicolumn{5}{|c|}{ Placebo } \\
\hline $\begin{array}{c}\text { Subjects } \\
n\end{array}$ & $\begin{array}{c}\text { Grade } 3 \\
\text { AE }\end{array}$ & $\begin{array}{c}\text { Grade } 4 \\
\text { AE }\end{array}$ & $\begin{array}{c}\text { Serious } \\
\text { AE }\end{array}$ & $\begin{array}{l}\text { AE leading to } \\
\text { treatment DC }\end{array}$ & $\begin{array}{c}\text { Subjects } \\
n\end{array}$ & $\begin{array}{c}\text { Grade } 3 \\
\text { AE }\end{array}$ & $\begin{array}{c}\text { Grade } 4 \\
\text { AE }\end{array}$ & $\begin{array}{c}\text { Serious } \\
\text { AE }\end{array}$ & $\begin{array}{l}\text { AE leading to } \\
\text { treatment } D C\end{array}$ \\
\hline
\end{tabular}

\begin{tabular}{|c|c|c|c|c|c|c|c|c|c|c|}
\hline & $\mathbf{n}$ & AE & AE & AE & treatment DC & $\mathbf{n}$ & AE & AE & AE & treatment DC \\
\hline \multicolumn{11}{|c|}{$\begin{array}{l}\text { Time since } \\
\text { diagnosis years }\end{array}$} \\
\hline$<1$ & 281 & 23.5 & 3.2 & 19.9 & 8.5 & 282 & 22.7 & 4.6 & 19.9 & 8.5 \\
\hline$>2$ & 169 & 26.6 & 3.6 & 21.3 & 13.6 & 177 & 29.9 & 6.2 & 26.6 & 9.0 \\
\hline \multicolumn{11}{|l|}{ FVC \% pred } \\
\hline$<65$ & 205 & 25.4 & 5.4 & 23.9 & 15.1 & 224 & 29.0 & 6.3 & 27.7 & 11.2 \\
\hline \multicolumn{11}{|l|}{ D Lco \% pred } \\
\hline$<40$ & 207 & 28.5 & 3.4 & 23.7 & 14.0 & 211 & 32.2 & 9.5 & 33.6 & 12.8 \\
\hline $40-<50$ & 229 & 25.3 & 5.2 & 23.1 & 12.7 & 230 & 22.6 & 4.8 & 20.4 & 8.3 \\
\hline$\geqslant 50$ & 187 & 19.3 & 1.6 & 13.9 & 9.1 & 181 & 16.6 & 1.7 & 11.6 & 4.4 \\
\hline \multicolumn{11}{|l|}{ 6MWD m } \\
\hline$<350$ & 168 & 33.3 & 5.4 & 26.8 & 13.7 & 141 & 33.3 & 11.3 & 34.8 & 14.9 \\
\hline Yes & 155 & 29.0 & 5.2 & 25.8 & 11.0 & 150 & 36.0 & 9.3 & 34.0 & 12.0 \\
\hline No & 468 & 23.1 & 3.0 & 18.8 & 12.4 & 474 & 20.3 & 4.2 & 18.6 & 7.6 \\
\hline
\end{tabular}

Data are presented as \% of patients in each stratum based on events occurring between the first dose and 28 days after the last dose of study drug, unless otherwise stated. AE: adverse event; DC: discontinuation; FVC: forced vital capacity; DLCo: diffusing capacity of the lung for carbon monoxide; 6MWD: 6-min walk distance. 
The results of our study should be interpreted in the context of certain limitations. First, while the pooled analyses of efficacy outcomes were pre-specified prior to completion of the ASCEND trial, the analysis plan was written after the results of the CAPACITY studies were known. We note, however, that the primary objective of the pooled analyses was to obtain the most stable estimates of the magnitude of the treatment effect in patients with IPF; inferential statistics were reported for the limited purpose of informing assessments of the variability of the treatment effect. Second, certain aspects of the ASCEND study design and eligibility criteria were modified relative to the CAPACITY studies to decrease heterogeneity and enrol patients with a higher risk of disease progression. Despite these modifications, baseline characteristics among patients in the ASCEND and CAPACITY studies were quite similar, and the observed magnitude of treatment effect at 1 year was consistent across studies. Finally, baseline measures of disease status in the pooled population were consistent with mild-to-moderate physiologic impairment; accordingly, the extent to which our findings are generalisable to patients with more severe physiologic impairment or comorbidities that mandated exclusion from the phase 3 trials is not known.

In conclusion, our results provide the most stable and robust estimates to date of the magnitude of the treatment effect of pirfenidone on multiple measures of IPF disease progression. These findings provide further evidence to support the clinically meaningful treatment benefit and acceptable safety profile of pirfenidone in patients with IPF.

\section{Acknowledgements}

The authors thank Kenneth Glasscock (InterMune, Brisbane, CA, USA) for medical writing and editorial assistance, and Gary Koch (University of North Carolina at Chapel Hill, Chapel Hill, NC, USA) for statistical consultation. The authors also thank the patients and participating staff members at all study sites.

\section{References}

1 Noble PW, Albera C, Bradford WZ, et al. Pirfenidone in patients with idiopathic pulmonary fibrosis (CAPACITY): two randomised trials. Lancet 2011; 377: 1760-1769.

2 King TE Jr, Bradford WZ, Castro-Bernardini S, et al. A phase 3 trial of pirfenidone in patients with idiopathic pulmonary fibrosis. N Engl J Med 2014; 370: 2083-2092.

3 Taniguchi H, Ebina M, Kondoh Y, et al. Pirfenidone in idiopathic pulmonary fibrosis. Eur Respir J 2010; 35: 821-829.

4 Azuma A, Nukiwa T, Tsuboi E, et al. Double-blind, placebo-controlled trial of pirfenidone in patients with idiopathic pulmonary fibrosis. Am J Respir Crit Care Med 2005; 171: 1040-1047.

5 Spagnolo P, Del Giovane C, Luppi F, et al. Non-steroid agents for idiopathic pulmonary fibrosis. Cochrane Database Syst Rev 2010; 9: CD003134.

6 Raghu G, Collard HR, Egan JJ, et al. An official ATS/ERS/JRS/ALAT statement: idiopathic pulmonary fibrosis: evidence-based guidelines for diagnosis and management. Am J Respir Crit Care Med 2011; 183: 788-724.

7 Lachin MJ. Worst-rank score analysis with informatively missing observations in clinical trials. Control Clin Trials 1999; 20: 408-422.

8 Finkelstein DM, Schoenfeld DA. Combining mortality and longitudinal measures in clinical trials. Stat Med 1999; 18: 1341-1354.

9 Martinez FJ, Safrin S, Weycker D, et al. The clinical course of patients with idiopathic pulmonary fibrosis. Ann Intern Med 2005; 142: 963-967.

10 Higgins JPT, Green S, eds. Cochrane Handbook for Systematic Reviews of Interventions Version 5.1.0 [updated March 2011]. The Cochrane Collaboration 2011. www.cochrane-handbook.org Date last accessed: August 25, 2015.

11 Roetzheim RG, Freund KM, Corle DK, et al. Analysis of combined data from heterogeneous study designs: an applied example from the patient navigation research program. Clin Trials 2012; 9: 176-187. 